\title{
High Angle Annular Dark Field Imaging on and away from the Pole
}

\author{
A. Bleloch*, P.Wang**, U. Falke* and P. Goodhew** \\ *SuperSTEM Laboratory, CLRC, Daresbury WA4 4AD, UK. \\ ** Materials Science and Engineering, University of Liverpool, L69 3GH, UK
}

High angle annular dark field (HAADF) imaging has proved to be a remarkably effective tool in elucidating material structures at the atomic scale in the scanning transmission electron microscope (STEM). It has the additional advantage of being compatible with simultaneous electron energy loss spectroscopy (EELS). A great deal of effort has been expended on understanding the imaging process in the usual orientation which is with the optic axis of the probe aligned with a pole of the sample [1,2]. Figure 1 shows an example of such an image of a ruthenia nanorod [3]. Also shown is the profile across the edge of the crystal. This is convincing evidence of the small probe size attainable with aberration correction though assigning the probe size to the quarter to three quarters width of this profile would neglect two important effects. First, the broadening caused by the extent of the sample along the beam direction and second any non-linear signal dependence on probe position caused by, say, channelling. The first would broaden and the second narrow the apparent probe width.

Here we also present some initial results exploring HAADF imaging as a crystal is tilted away from the pole and as a function of probe size - and hence describe, from a different perspective an advantage of controlling electron optical aberrations. In particular, we are interested in describing the imaging of polycrystalline samples. In high-resolution transmission electron microscopy (HRTEM), fringes are present in almost every grain of a thin polycrystalline sample. This is readily understood from the geometry of the Ewald sphere construction. In most cases, the thin sample has caused the reciprocal lattice spots to become extended along a line along (or at a small angle to) the optic axis. Therefore, because of the large wavevector of the illuminating electron wave compared to typical reciprocal lattice spacings, there is a very high probability that the Ewald sphere will intersect extended reciprocal lattice "spots" within the angular range of the objective aperture and therefore produce fringes in the image.

No such simple explanation exists in the case of HAADF imaging. As a first attempt to understand the probability of fringe visibility we began by measuring fringe contrast as a function of angular misalignment from the pole. Fig 2 shows that fringe contrast is visible up to $1.4^{\circ}$ away from pole. What fraction of all possible crystal orientations will show contrast? This depends on the resolution of the probe - the smaller the probe, the finer the spacing that can be imaged. The fraction can be computed by integrating over all solid angles that fall within a given angle (say $1^{\circ}$ ) of a all planes that can be imaged with a probe of given size. Fig 3 shows this fraction of angles for which fringe contrast should be visible as a function of allowed tilt from the pole for different probe sizes. Fig. 4 is a HAADF image of a polycrystalline silicon sample with a grain size in the region of 50nm. The image was obtained by scanning 5 successive images and adding them together. No re-registration of the images was performed because the microscope stability is sufficient to maintain the atomic columns within a small fraction of an angstrom for the duration of the scans. Despite the integration, the contrast in the polycrystalline region remains low, but, as expected from the analysis above, most grains exhibit some fringes. 

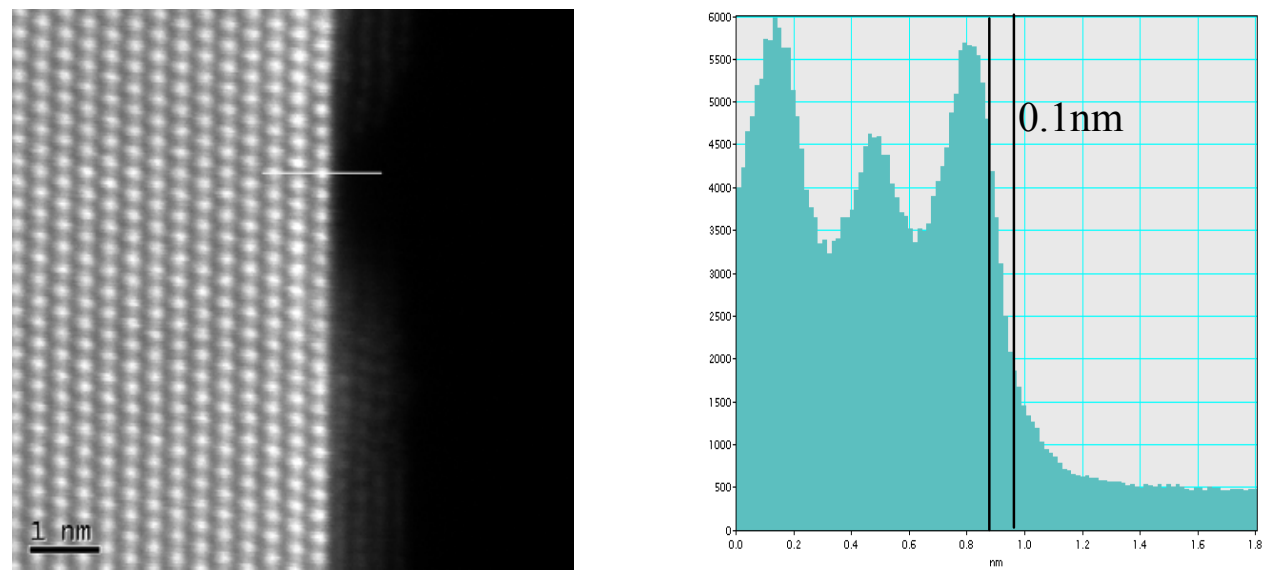

Fig. 1 HAADF image of Ruthenia with edge profile [5].
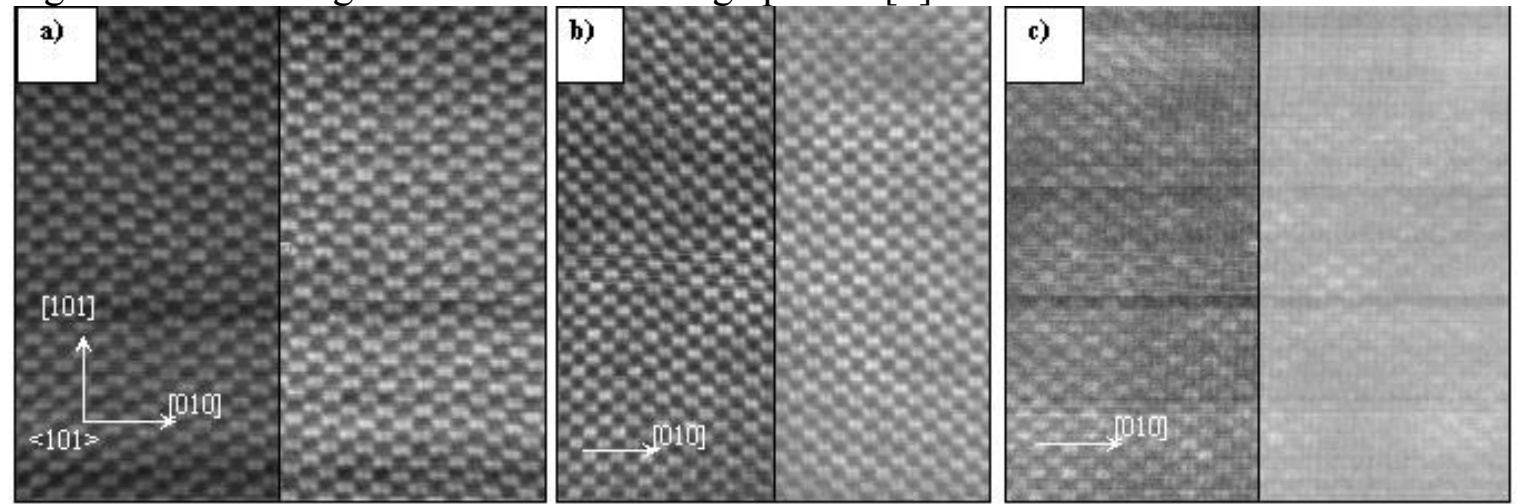

Fig. 2 HAADF Silicon lattice images. a) On 110 pole b) $0.94^{\circ}$ and c) $1.4^{\circ}$. Left half is contrast stretched and right half are all with the same contrast
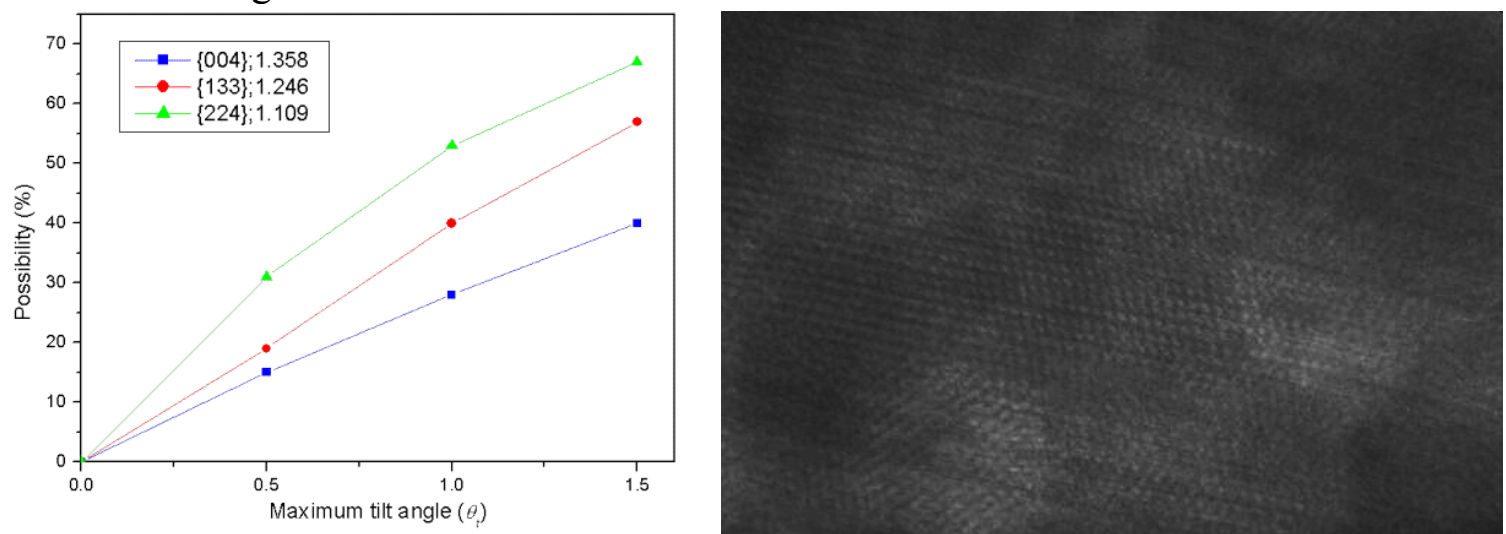

Fig. 3 Probability of fringe visibility as a Fig. 4. HAADF of polycrystalline silicon. function of tilt and for 3 probe sizes

References and acknowledgements:

1. E. J. Kirkland, R. F. Loane and J. Silcox, Ultramicroscopy, 23, (1987), 77-96

2. P. D. Nellist and S. J. Pennycook, Ultramicroscopy, 78, (1999), 111-124

3. Ducati C. et al. Proceedings of the 13th EUREM Antwerp, August 22-27, 2004, Vol II, MS03.O1

4. P. Wang et al. To be submitted to ultramicroscopy

5. Sample from C. Ducati and P. Midgeley, Cambridge University, Materials

6. We would like to thank the EPSRC, CLRC Daresbury, EMAG (IOP), and the RMS 